\begin{tabular}{|c|l|}
\hline Title & Ground state of a spin 1/2 charged particle in an even dimensional magnetic field \\
\hline Author(s) & Ogurisu, O. \\
\hline Citation & Hokkaido University Preprint Series in Mathematics, 180, 1-9 \\
\hline Issue Date & 1993-01 \\
\hline DOI & 10.14943/83324 \\
\hline Doc URL & http://hdl.handle.net/2115/68926 \\
\hline Type & bulletin (article) \\
\hline File Information & pre180.pdf \\
\hline
\end{tabular}

Instructions for use 
GROUND STATE OF A SPIN $1 / 2$ CHARGED PARTICLE IN AN EVEN

DIMENSIONAL MAGNETIC FIELD

\author{
Osamu Ogurisu
}

Series $\sharp 180$. January 1993 


\section{HOKKAIDO UNIVERSITY}

\section{PREPRINT SERIES IN MATHEMATICS}

\# 153: Y. Watatani, Lattices of intermediate subfactors, 33 pages. 1992.

154: T. Ozawa, On critical cases of Sobolev inequalities, 11 pages. 1992.

$\sharp 155: \quad$ M. Ohnuma, M. Sato, Singular degenerate parabolic equations with applications to geometric evolutions, 20 pages. 1992.

\#156: S. Izumiya, Perestroikas of optical wave fronts and graphlike Legendrian unfoldings, 13 pages. 1992.

$\sharp 157$ : A. Arai, Momentum operators with gauge potentials, local quantization of magnetic flux, and representation of canonical commutation relations, 11 pages. 1992.

$\sharp 158$ : S. Izumiya, W.L. Marar, The Euler number of a topologically stable singular surface in a 3-manifold, 11 pages. 1992.

$\sharp 159$ : T. Hibi, Cohen-Macaulay types of Cohen-Macaulay complexes, 26 pages. 1992.

$\$ 160$ : A. Arai, Properties of the Dirac-Weyl operator with a strongly singular gauge potential, 26 pages. 1992.

$\sharp$ 161: A. Arai, Dirac operators in Boson-Fermion Fock spaces and supersymmetric quantum field theory, 30 pages. 1992.

\$162: S. Albeverio, K. Iwata, T. Kolsrud, Random parallel transport on surfaces of finite type, and relations to homotopy, 8 pages. 1992.

$\sharp 163$ : S. Albeverio, K. Iwata, T. Kolsrud, Moments of random fields over a family of elliptic curves, and modular forms, 9 pages. 1992.

\# 164: Y. Giga, M. Sato, Neumann problem for singular degenerate parabolic equations, 12 pages. 1992.

\# 165: J. Wierzbicki, Y. Watatani, Commuting squares and relative entropy for two subfactors, 18 pages. 1992.

$\sharp 166$ : Y. Okabe, A new algorithm driven from the view-point of the fluctuation-dissipation theorem in the theory of $\mathrm{KM}_{2} \mathrm{O}$-Langevin equations, 13 pages. 1992.

$\sharp 167$ : Y. Okabe, H. Mano and Y. Itoh, Random collision model for interacting populations of two species and its strong law of large numbers, 14 pages. 1992.

$\sharp 168$ : A. Inoie, On the equations of stationary precesses with divergent diffusion coefficients, 25 pages. 1992.

\$169: T. Ozawa, Remarks on quadratic nonlinear Schrödinger equations, 19 pages. 1992.

$\sharp 170$ : T. Fukni, Y. Giga, Motion of a graph by nonsmooth weighted curvature, 11 pages. 1992.

$\sharp 171$ J J. Inoue, T. Nakazi, Finite dimensional solution sets of extremal problems in $\mathrm{H}^{1}, 10$ pages. 1992.

$\sharp 172$ : S. Izumiya, A characterization of complete integrability for partial differential equations of first order, 6 pages. 1992.

$\sharp 173$ : T. Suwa, Unfoldings of codimension one complex analytic foliation singularities, 49 pages. 1992.

$\sharp 174: \quad$ T. Ozawa, Wave propagation in even dimensional spaces, 15 pages. 1992.

$\sharp 175: \quad$ S. Izumiya, Systems of Clairaut type, 7 pages. 1992.

\#176: A. Hoshiga, The initial value problems for quasi-linear wave equations in two space dimensions with small data, 25 pages. 1992.

$\sharp 177$ : K. Sugano, On bicommutators of modules over H-separable extension rings III, 9 pages. 1993.

$\sharp 178$ : T. Nakazi, Toeplitz operators and weighted norm inequalities, 17 pages. 1993.

$\sharp 179: \quad O$. Ogurisu, Existence and structure of infinitely degenerate zero-energy ground states of a Wess-Zumino type model in supersymmetric quantum mechanics, 26 pages. 1993. 


\title{
GROUND STATE OF A SPIN 1/2 CHARGED PARTICLE IN AN EVEN DIMENSIONAL MAGNETIC FIELD
}

\author{
OSAMU OGURISU \\ Department of Mathematics, Hokkaido University \\ Sapporo 060, Japan
}

12, January 1993

\begin{abstract}
We investigate the ground state structure of the Schrödinger operator (Pauli Hamiltonian) $H$ with a magnetic field $b$ for a spin $1 / 2$ particle in $\mathbb{R}^{2 d} \cong \mathbb{C}^{d}$. We consider the case where $b$ is given by the complex exterior derivative of a function $W$ on $\mathbb{C}^{d}$ of the form $b=i(\bar{\partial}+\partial)(\bar{\partial}-\partial) W$. We found that $\operatorname{dim} \operatorname{ker} H$ is related to the asymptotic behavior of $W$ at infinity. More precisely, if there exists a constant $C \in \mathbb{R}$ such that $W(z) \sim-C \log |z|$ as $z \rightarrow \infty$, then $\operatorname{dim} \operatorname{ker} H$ is equal to the number of all monomials $f$ in $d$ variables such that the degree of $f$ is smaller than $|C|-d$. Moreover we clarify the structure of ker $H$.
\end{abstract}




\section{INTRODUCTION}

We investigate the ground state structure of the Schrödinger operator $H$ with a magnetic field for a spin $1 / 2$ particle in the $2 d$-dimensional Euclidean space $\mathbb{R}^{2 d},(d \geq 1)$. Let $r=2^{d}$ and $\gamma^{i}$ s be $r \times r$ Hermitian matrices (the so-called Dirac matrices) satisfying

$$
\gamma^{i} \gamma^{j}+\gamma^{j} \gamma^{i}=2 \delta^{i j}, \quad i, j=1, \cdots, 2 d
$$

where $\delta^{i j}$ is the Kronecker delta. Let $a(x)=\sum_{i=1}^{2 d} a_{i}(x) d x^{i}$ be a real 1-form on $\mathbb{R}^{2 d}$, which is called a vector potential. Define the Dirac operator $\not D(a)$ by

$$
\not D(a)=\sum_{k=1}^{2 d} \gamma^{k}\left(-i \frac{\partial}{\partial x^{k}}-a_{k}(x)\right)
$$

acting in $L^{2}\left(\mathbb{R}^{2 d}\right) \otimes \mathbb{C}^{r}$. The Schrödinger operator (the Pauli Hamiltonian) $H$ we are going to study is given by

$$
H=\not D(a)^{2}
$$

The relation (2.1) is the fundamental relation of the Clifford algebra associated with $\mathbb{R}^{2 d}$. The representation space of the Clifford algebra in this formulation is $\mathbb{C}^{r}$. The 2-form

$$
b=d a
$$

is called the magnetic field. Throughout this paper, we assume that $a_{i}$ 's are $C^{\infty}$-functions. Then $\not D(a)$ and $H$ are essentially self-adjoint operators on $C_{0}^{\infty}\left(\mathbb{R}^{2 d}\right) \otimes \mathbb{C}^{r}$ (see [1]).

Shigekawa [2] studied the relation between spectral properties of $H$ and the asymptotic behavior of $b$ at infinity in any finite dimensions. In particular, in the even dimensional case, identifying $\mathbb{R}^{2 d}$ with $\mathbb{C}^{d}$ and assuming that

(A) there exists a function $W \in \mathbb{C}^{\infty}\left(\mathbb{C}^{d} \rightarrow \mathbb{R}\right)$ such that $b=i d(\bar{\partial}-\partial) W$,

where $\partial$ and $\bar{\partial}$ are the complex exterior differential and its conjugation on $\mathbb{C}^{d}$, respectively, he proved that it has been proved that 0 is an infinitely degenerate eigenvalue of $H$ unless $b(z)$ goes to 0 as $z \rightarrow \infty$ so rapidly (see [2]).

In this paper, under the same assumption (A), we prove that dim ker $H$ is related to the asymptotic behavior of $W$ at infinity. More precisely, if there exists a constant $C \in \mathbb{R}$ such that $W(z) \sim-C \log |z|$ as $z \rightarrow \infty$, then $\operatorname{dim} \operatorname{ker} H$ is equal to the number of all monomials 
$f$ in $d$ variables such that the degree of $f$ is smaller than $|C|-d$. Moreover we clarify the structure of $\operatorname{ker} H$.

In Sec.II we give an expression of the Dirac operator $\not D(a)$ as an operator in a space of differential forms. In Sec.III we prove the main theorem (Theorem 3.1) and give several remarks.

\section{AN Expression of the DiRac Operator}

In this section, we realize the Dirac operator $\not D(a)$ as an operator on a space of differential forms (see [2]). From now on, we work in the space $\mathbb{C}^{d}$. For $z=\left(z^{1}, \ldots, z^{d}\right) \in \mathbb{C}^{d}$, we write $z^{k}=x^{k}+i y^{k}, x^{k}, y^{k} \in \mathbb{R}$ and as usual we define tangent and cotangent vectors by

$$
\begin{aligned}
\frac{\partial}{\partial z^{k}} & =\frac{1}{2}\left(\frac{\partial}{\partial x^{k}}-i \frac{\partial}{\partial y^{k}}\right) \\
\frac{\partial}{\partial \bar{z}^{k}} & =\frac{1}{2}\left(\frac{\partial}{\partial x^{k}}+i \frac{\partial}{\partial y^{k}}\right) \\
d z^{k} & =d x^{k}+i d y^{k} \\
d \bar{z}^{k} & =d x^{k}-i d y^{k}
\end{aligned}
$$

We denote by $\wedge^{0, q}\left(T^{*} \mathbb{C}^{d}\right)$ the space of all $(0, q)$-type differential forms. Any element $\omega \in$ $\wedge^{0, q}\left(T^{*} \mathbb{C}^{d}\right)$ is expressed as

$$
\omega=\sum_{I} \omega_{I} d \bar{z}^{I}
$$

where $I=\left\{1 \leq i_{1}<\cdots<i_{q} \leq d\right\}$ and $d \bar{z}^{I}=d \bar{z}^{i_{1}} \wedge \cdots \wedge d \bar{z}^{i_{q}}$.

We take the exterior algebra $\wedge^{0, *}\left(\mathbb{C}^{d}\right)^{*}=\oplus_{q=0}^{d} \wedge^{0, q}\left(\mathbb{C}^{d}\right)^{*}$ as a representation space of Clifford algebra. We define an operator $\operatorname{ext}\left(d \bar{z}^{i}\right)$ on $\wedge^{0, *}\left(\mathbb{C}^{d}\right)^{*}$ by

$$
\operatorname{ext}\left(d \bar{z}^{i}\right) \eta=d \bar{z}^{i} \wedge \eta, \quad \eta \in \wedge^{0, *}\left(\mathbb{C}^{d}\right)^{*}
$$

Let $\operatorname{int}\left(d z^{i}\right)$ be the adjoint operator of $\operatorname{ext}\left(d \bar{z}^{i}\right)$. Then, we have that for $I=\left\{i_{1}, \ldots, i_{q}\right\}$

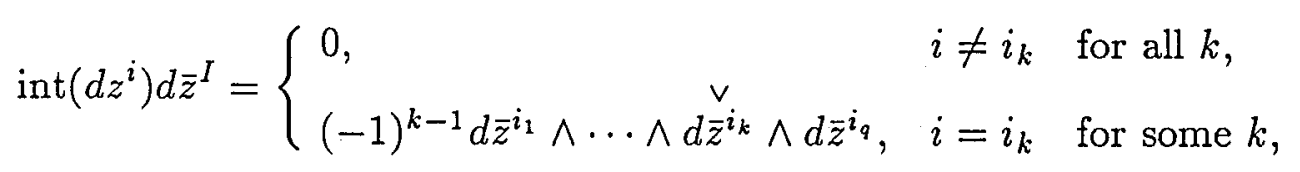


where $d \bar{z}^{i_{k}}$ means that $d \bar{z}^{i_{k}}$ is removed. Let

$$
\begin{aligned}
& \gamma^{2 i-1}=i\left(\operatorname{ext}\left(d \bar{z}^{i}\right)-\operatorname{int}\left(d z^{i}\right)\right) \\
& \gamma^{2 i}=-\left(\operatorname{ext}\left(d \bar{z}^{i}\right)+\operatorname{int}\left(d z^{i}\right)\right)
\end{aligned}
$$

We denote by $[\cdot, \cdot]_{+}$the anti-commutator. The well-known fact that

$$
\begin{aligned}
& {\left[\operatorname{ext}\left(d \bar{z}^{i}\right), \operatorname{ext}\left(d \bar{z}^{j}\right)\right]_{+}=\left[\operatorname{int}\left(d z^{i}\right), \operatorname{int}\left(d z^{j}\right)\right]_{+}=0,} \\
& {\left[\operatorname{ext}\left(d \bar{z}^{i}\right), \operatorname{int}\left(d z^{j}\right)\right]_{+}=\delta^{i j}}
\end{aligned}
$$

implies that $\left[\gamma^{i}, \gamma^{j}\right]_{+}=2 \delta^{i j}$, which is the fundamental relation (2.1) of Clifford algebra.

As usual, the complex exterior differential $\bar{\partial}$ on $\wedge^{0, *}\left(T^{*} \mathbb{C}^{d}\right)=\oplus_{q=0}^{d} \wedge^{0, q}\left(T^{*} \mathbb{C}^{d}\right)$ is given by

$$
\bar{\partial} \omega=\sum_{I} \sum_{i=1}^{d} \frac{\partial \omega_{I}}{\partial \bar{z}^{i}} d \bar{z}^{i} \wedge d \bar{z}^{I}
$$

Now we identify $\wedge^{0, *}\left(T^{*} \mathbb{C}^{d}\right)$ and $C^{\infty}\left(\mathbb{C}^{d}\right) \otimes \wedge^{0, *}\left(\mathbb{C}^{d}\right)^{*}$, so $\bar{\partial}$ is expressed as follows:

$$
\bar{\partial}=\sum_{i=1}^{d} \operatorname{ext}\left(d \bar{z}^{i}\right) \frac{\partial}{\partial \bar{z}^{i}} .
$$

We regard $\bar{\partial}$ as an operator in the Hilbert space $L^{2}\left(\wedge^{0, *}\left(T^{*} \mathbb{C}^{d}\right)\right)=L^{2}\left(\mathbb{C}^{d}\right) \otimes \wedge^{0, *}\left(\mathbb{C}^{d}\right)^{*}$, the space of all square integrable sections with respect to the Lebesgue measure on $\mathbb{C}^{d}$. The adjoint operator $\bar{\partial}^{*}$ of $\bar{\partial}$ is expressed as

$$
\bar{\partial}^{*}=-\sum_{i=1}^{d} \operatorname{int}\left(d z^{i}\right) \frac{\partial}{\partial z^{i}}
$$

Next we realize the Dirac operator $\not D(a)$ as an operator in $L^{2}\left(\mathbb{C}^{d}\right) \otimes \wedge^{0, *}\left(\mathbb{C}^{d}\right)^{*}$. We assume the following.

(H). There exists a function $W \in C^{\infty}\left(\mathbb{C}^{d} \rightarrow \mathbb{R}\right)$ such that $a=i(\bar{\partial}-\partial) W$.

We remark that, if the real 2 -form $b=d a$ is of type $(1,1)$, then there exists a $W \in$ $C^{\infty}\left(\mathbb{C}^{d} \rightarrow \mathbb{R}\right)$ such that $a=i(\bar{\partial}-\partial) W($ see $[3$, Lemma II.2.15]), and that in the real 2dimensional case, any 2 -form is always of type $(1,1)$. 
We take a $W$ as in $(\mathrm{H})$ and fix it. Define an operator $\bar{\partial}_{W}$ in $L^{2}\left(\mathbb{C}^{d}\right) \otimes \wedge^{0, *}\left(\mathbb{C}^{d}\right)^{*}$ by

$$
\bar{\partial}_{W}=\bar{\partial}+\operatorname{ext}(\bar{\partial} W)=\sum_{i=1}^{d} \operatorname{ext}\left(d \bar{z}^{i}\right)\left(\frac{\partial}{\partial \bar{z}^{i}}+\frac{\partial W}{\partial \bar{z}^{i}}\right) .
$$

Then the adjoint operator of $\bar{\partial}_{W}$ is given by

$$
\bar{\partial}_{W}^{*}=\bar{\partial}^{*}+\operatorname{int}(\partial W)=\sum_{i=1}^{d} \operatorname{int}\left(d z^{i}\right)\left(-\frac{\partial}{\partial z^{i}}+\frac{\partial W}{\partial z^{i}}\right)
$$

By a straightforward computation, we can show that

$$
2\left(\bar{\partial}_{W}+\bar{\partial}_{W}^{*}\right)=\not D(a)
$$

Thus the Dirac operator is realized as an operator in a space of differential forms.

\section{MAIN THEOREM}

For a real number $C$, we denote by $N_{d}(C)$ the number of all monomials $f$ in $d$ variables such that the degree of $f$ is smaller than $|C|-d$ :

$$
N_{d}(C)=\sharp\left\{\left(\alpha_{1}, \ldots, \alpha_{d}\right) ; 0 \leq \alpha_{1}+\cdots+\alpha_{d}<|C|-d, \alpha_{j} \in \mathbb{N} \cup\{0\}, j=1, \ldots, d\right\} .
$$

The following theorem is the main theorem in this paper.

Theorem 3.1. Assume that the hypothesis $(\mathbf{H})$ in Sec.II holds and that the limit

$$
C=-\lim _{z \rightarrow \infty} \frac{W(z)}{\log |z|}
$$

exists. Then

$$
\operatorname{dim} \operatorname{ker} H=N_{d}(C)
$$

In particular, if $W$ is bounded then $\operatorname{dim} \operatorname{ker} H=0$.

Remark. We emphasize that $\operatorname{dim} \operatorname{ker} H$ is determined by the behavior of $W(z)$ at $z \sim \infty$. Note that, in [2], the relation between spectral properties of $H$ and the asymptotic behavior of $b$ at infinity is treated.

Proof. First, we treat the case where $C \geq 0$. We denote by $m$ the Lebesgue measure on $\mathbb{C}^{d}$ :

$$
m(d z)=d x^{1} d y^{1} \cdots d x^{d} d y^{d}
$$


We consider a unitary operator $U: L^{2}\left(\mathbb{C}^{d}\right) \otimes \wedge^{0, *}\left(\mathbb{C}^{d}\right)^{*} \rightarrow L^{2}\left(\mathbb{C}^{d} ; e^{-2 W} m\right) \otimes \wedge^{0, *}\left(\mathbb{C}^{d}\right)^{*}$ given by

$$
U \omega=e^{W} \omega
$$

Then we have that $U \bar{\partial}_{W} U^{*}=\bar{\partial}$. Let $\bar{\partial}^{*}$ be the adjoint operator of $\bar{\partial}$ with respect to the measure $e^{-2 W} m$, and we put $\hat{H}=U H U^{*}$. Since $\bar{\partial}^{2}=\left(\bar{\partial}^{*}\right)^{2}=0$, we have

$$
(\hat{H} \omega, \omega)=4 \int_{\mathbb{C}^{d}}\left(|\bar{\partial} \omega(z)|^{2}+\left|\bar{\partial}^{*} \omega(z)\right|^{2}\right) e^{-2 W(z)} m(d z)
$$

Suppose that $\omega \in \operatorname{ker} \hat{H}$. We show that $\omega$ is of type $(0, d)$. We can write $\omega=\omega_{1}+\omega_{2}$, where

$$
\begin{aligned}
& \omega_{1} \in L^{2}\left(\mathbb{C}^{d} ; e^{-2 W} m\right) \otimes{\underset{q=0}{d-1} \wedge^{0, q}\left(\mathbb{C}^{d}\right)^{*},}_{\omega_{2} \in L^{2}\left(\mathbb{C}^{d} ; e^{-2 W} m\right) \otimes \wedge^{0, d}\left(\mathbb{C}^{d}\right)^{*}} .
\end{aligned}
$$

The first term $\omega_{1}$ can be expressed as

$$
\omega_{1}(z)=\sum_{I} \omega_{I}(z) d \bar{z}^{I}
$$

where the summation is over all proper subsets $I$ of $\{1,2, \ldots, d\}$ and $\omega_{I} \in L^{2}\left(\mathbb{C}^{d} ; e^{-2 W} m\right)$. Since $\bar{\partial} \omega=\bar{\partial} \omega_{1}$, we see

$$
\bar{\partial} \omega(z)=\sum_{I} \sum_{i \notin I} \frac{\partial \omega_{I}(z)}{\partial \bar{z}^{i}} d \bar{z}^{i} \wedge d \bar{z}^{I}
$$

By (3.1) we obtain

$$
0=\int_{\mathbb{C}^{d}}|\bar{\partial} \omega(z)|^{2} e^{-2 W(z)} m(d z)=\sum_{I} \sum_{i \notin I} \int_{\mathbb{C}^{d}}\left|\frac{\partial \omega_{I}(z)}{\partial \bar{z}^{i}}\right|^{2} e^{-2 W(z)} m(d z)
$$

Since $I$ is a proper subset of $\{1,2, \ldots, d\}$, we may assume that $d \notin I$ without loss of generality. Then we have

$$
\int_{\mathbb{C}^{d}}\left|\frac{\partial \omega_{I}(z)}{\partial \bar{z}^{d}}\right|^{2} e^{-2 W(z)} m(d z)=0
$$

Since $e^{-2 W(z)}>0$, we obtain

$$
\frac{\partial \omega_{I}(z)}{\partial \bar{z}^{d}}=0 \text { on } \quad \mathbb{C}^{d}
$$


This means that $\omega_{I}$ is an entire function in $z^{d}$. On the other hand, $\omega_{I}$ must be in $L^{2}\left(\mathbb{C}^{d} ; e^{-2 W} m\right)$. Since $W$ is bounded from above, $\omega_{I} \in L^{2}\left(\mathbb{C}^{d}\right)$. By Fubini's theorem, it holds that

$$
\int_{\mathbb{C}}\left|\omega_{I}\left(z_{0}, z^{d}\right)\right|^{2} d x^{d} d y^{d}<\infty
$$

for a.e. $z_{0}=\left(z^{1}, z^{2}, \ldots, z^{d-1}\right)$ with respect to the measure $d x^{1} d y^{1} \cdots d x^{d-1} d y^{d-1}$. Since $\omega_{I}\left(z_{0}, \cdot\right)$ is entire, $\omega_{I}\left(z_{0}, \cdot\right)$ must be equal to 0 . Thus we see $\omega_{I}=0$ a.e. with respect to $m$ on $\mathbb{C}^{d}$. Consequently we have that $\omega_{1}=0$. Hence $\omega=\omega_{2}$.

Since $\operatorname{ker} \hat{H}=\operatorname{ker} \not D(a) U^{*}$, it suffices to compute the dimension of $\operatorname{ker} \not D(a) U^{*}$. We can write

$$
\left(U^{*} \omega\right)(z)=\tilde{\omega}(z) d \bar{z}^{1} \wedge \cdots \wedge d \bar{z}^{d}
$$

where $\tilde{\omega} \in L^{2}\left(\mathbb{C}^{d}\right)$. Since $\bar{\partial}_{W} U^{*} \omega=0$, it follows that

$$
\not D(a) U^{*} \omega=2 \bar{\partial}_{W}^{*} U^{*} \omega=2 \sum_{i=1}^{d}\left(-\frac{\partial \tilde{\omega}}{\partial z^{i}}+\frac{\partial W}{\partial z^{i}} \tilde{\omega}\right)(-1)^{i-1} d \bar{z}^{1} \wedge \cdots \wedge d \bar{z}^{i} \wedge \cdots \wedge d \bar{z}^{d}
$$

Therefore the equality $\not D(a) U^{*} \omega=0$ implies

$$
-\frac{\partial \tilde{\omega}}{\partial z^{i}}+\frac{\partial W}{\partial z^{i}} \tilde{\omega}=0, \quad i=0, \ldots, d .
$$

Let $f=e^{-W} \tilde{\omega}$. We obtain the equations

$$
\frac{\partial f}{\partial z^{i}}=0, \quad i=0, \ldots, d
$$

This means that $f$ is an anti-holomorphic function in each $z^{i}, i=1, \ldots, d$. By the assumption, we have that

$$
e^{W(z)} \sim \frac{1}{|z|^{C}} \text { as } \quad|z| \rightarrow \infty
$$

Hence $f e^{W} \in L^{2}\left(\mathbb{C}^{d}\right)$ if and only if $f$ is a polynomial in $\bar{z}^{1}, \cdots, \bar{z}^{d}$ such that the degree of $f$ is smaller than $C-d$. Therefore, $\operatorname{dim} \operatorname{ker} \hat{H}$ is equal to $N_{d}(C)$.

Next, we consider the case where $C<0$. A unitary operator $U: L^{2}\left(\mathbb{C}^{d}\right) \otimes \wedge^{0, *}\left(\mathbb{C}^{d}\right)^{*} \rightarrow$ $L^{2}\left(\mathbb{C}^{d} ; e^{2 W} m\right) \otimes \wedge^{0, *}\left(\mathbb{C}^{d}\right)^{*}$ is given by $U \omega=e^{-W} \omega$. Then we have that

$$
U \bar{\partial}_{W}^{*} U^{*}=\sum_{i=1}^{d} \operatorname{int}\left(d z^{i}\right) \frac{\partial}{\partial z^{i}}
$$


For $\omega \in \operatorname{ker} U H U^{*}$ we can write $\omega=\omega_{1}+\omega_{2}$, where $\omega_{1} \in L^{2}\left(\mathbb{C}^{d} ; e^{2 W} m\right) \otimes \wedge^{0,0}\left(\mathbb{C}^{d}\right)^{*}$ and $\omega_{2} \in L^{2}\left(\mathbb{C}^{d} ; e^{2 W} m\right) \otimes \oplus_{p=1}^{d} \wedge^{0, p}\left(\mathbb{C}^{d}\right)^{*}$. Then, in a way similar to the case where $C \geq 0$, we can obtain that $\omega=\omega_{1}$ and that $\operatorname{dim} \operatorname{ker} H$ is equal to $N_{d}(C)$. Thus we obtain the desired result.

From the proof of Theorem 3.1 we can also clarify the structure of ker $H$.

Corollary 3.2. Under the assumption of Theorem 3.1, the following holds: Let $C \geq 0$ (resp. $C<0$ ). Then each vector in $\operatorname{ker} H$ has the form;

$$
f e^{W} d \bar{z}^{1} \wedge \cdots \wedge d \bar{z}^{d} \quad\left(\text { resp. } f e^{-W}\right)
$$

Here, $f$ is a polynomial in $z^{1}, \ldots, z^{d}$ (resp. $\bar{z}^{1}, \ldots, \bar{z}^{d}$ ) such that the degree of $f$ is smaller than $|C|-d$.

In the rest of this section we give several remarks

Remark 3.3. In the 2-dimensional case, Aharonov and Casher has proved Theorem 3.1 in another context (see [4]).

Remark 3.4. In the special case where there exist constants $C \in \mathbb{R}$ and $R>0$ such that $W(z)=-C \log |z|$ for $|z|>R$, the magnetic field $b=i d(\bar{\partial}-\partial) W$ goes to 0 as $z \rightarrow \infty$. Then, by Theorem 2.2 in [2], the spectrum of $H$ is equal to $[0, \infty)$. Hence $H$ is not a Fredholm operator (see [5]).

Remark 3.5. We can state Theorem 3.1 in terms of index theory. Let

$$
\Gamma=(-i)^{d} \gamma^{1} \gamma^{2} \cdots \gamma^{2 d}
$$

We can easily check that $\Gamma$ is self-adjoint, $\sigma(\Gamma)=\sigma_{p}(\Gamma)=\{-1,1\}, \operatorname{ker}(\Gamma-1)=L^{2}\left(\mathbb{C}^{d}\right) \otimes$ $\oplus_{q}$ : even $\wedge^{0, q}\left(\mathbb{C}^{d}\right)^{*}$ and $\operatorname{ker}(\Gamma+1)=L^{2}\left(\mathbb{C}^{d}\right) \otimes \oplus_{q: \text { odd }} \wedge^{0, q}\left(\mathbb{C}^{d}\right)^{*}$, where we denote by $\sigma(\Gamma)$ and $\sigma_{p}(\Gamma)$ the spectrum and the point spectrum of $\Gamma$, respectively. Moreover, we see

$$
[\not D(a), \Gamma]_{+}=0
$$

Hence we can write

$$
\not D(a)=\left(\begin{array}{cc}
0 & A \\
A^{*} & 0
\end{array}\right)
$$


with $A$ being a densely defined closed linear operator from $\operatorname{ker}(\Gamma-1)$ to $\operatorname{ker}(\Gamma+1)$. As usual, we define the index of $A$ by $\operatorname{Ind}(A)=\operatorname{dim} \operatorname{ker} A-\operatorname{dim} \operatorname{ker} A^{*}$. By virtue of Corollary 3.2, we can verify that

$$
\operatorname{Ind}(A)= \begin{cases}N_{d}(C), & C<0 \text { or } d \text { is even } \\ -N_{d}(C), & \text { otherwise. }\end{cases}
$$

Acknowledgement. The author would like to thank Professor A. Arai for helpful conversations and encouragement.

\section{References.}

1. Chernoff, P. R., Essentially self-adjointness of powers of generators of hyperbolic equations, J. Funct. Anal. 12 (1973), 401-414.

2. Shigekawa, I., Spectral properties of Schrödinger operators with magnetic fields for a spin $1 / 2$ particle, J. Funct. Anal. 101 (1991), 255-285.

3. Wells, R. O., Differential Analysis on Complex Manifolds, Springer-Verlag, New YorkHeidelberg, 1980.

4. Aharonov, Y. and Casher, A., Ground state of a spin 1/2 charged particle in a twodimensional magnetic field, Phys. Rev. A 19 (1979), 2461-2462.

5. Ogurisu, O., Existence and structure of the infinitely degenerate zero-energy ground states of Wess-Zumino type model in supersymmetric quantum mechanics, to appear in J. Math. Phys. 